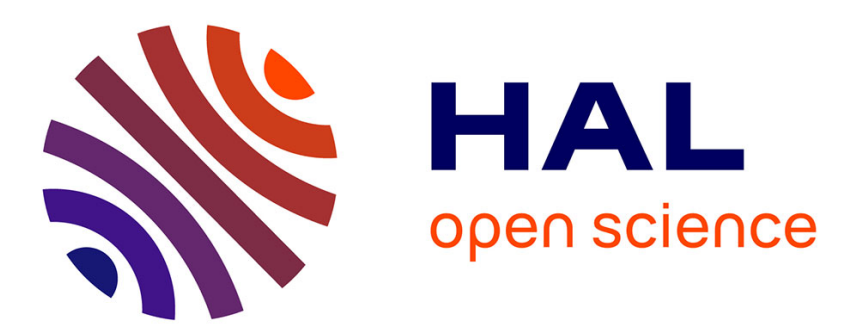

\title{
Calcul des fréquences localisées de vibration de surfaces libres et en présence d'une couche d'adsorbat par une méthode analytique très simple
}

D. Lagersie, M. Lannoo, L. Dobrzynski

\section{- To cite this version:}

D. Lagersie, M. Lannoo, L. Dobrzynski. Calcul des fréquences localisées de vibration de surfaces libres et en présence d'une couche d'adsorbat par une méthode analytique très simple. Journal de Physique, 1971, 32 (11-12), pp.963-971. 10.1051/jphys:019710032011-12096300 . jpa-00207197

\section{HAL Id: jpa-00207197 https://hal.science/jpa-00207197}

Submitted on 1 Jan 1971

HAL is a multi-disciplinary open access archive for the deposit and dissemination of scientific research documents, whether they are published or not. The documents may come from teaching and research institutions in France or abroad, or from public or private research centers.
L'archive ouverte pluridisciplinaire HAL, est destinée au dépôt et à la diffusion de documents scientifiques de niveau recherche, publiés ou non, émanant des établissements d'enseignement et de recherche français ou étrangers, des laboratoires publics ou privés. 


\title{
CALCUL DES FRÉQUENCES LOCALISÉES DE VIBRATION DE SURFACES LIBRES ET EN PRÉSENCE D'UNE COUCHE D'ADSORBAT PAR UNE MÉTHODE ANALYTIQUE TRÈS SIMPLE
}

\author{
D. LAGERSIE $(*)$, M. LANNOO et L. DOBRZYNSKI \\ Laboratoire de Physique des Solides (**), G. E. F. I. R. N., 3, rue François Baës. 59, Lille \\ (Reçu le 15 juillet 1971)
}

\begin{abstract}
Résumé. - Le but de ce travail est d'étudier les modes de vibration de surfaces (ondes de Rayleigh) par une méthode de moments associée à des modèles de variation de densité d'états à base de fonction delta, pour un cristal cubique simple monoatomique et dans deux cas :

- d'abord dans le cas de deux surfaces libres, en tenant compte des interactions entre premiers et seconds voisins, pour un modèle de cristal invariant par rotation et isotrope à lá limite des grandes longueurs d'ondes ;

- puis dans le cas d'une couche adsorbée d'atomes, en considérant que seule la masse des atomes de surface varie, pour un modèle simplifié de cristal invariant par translation avec interactions entre premiers voisins.

En comparant nos résultats avec ceux obtenus par un calcul détaillé par la méthode des déphasages, nous retrouvons les mêmes courbes de dispersion à $5 \%$ près dans les cas les moins favorables et souvent à $1 \%$ près, avec l'avantage d'une méthode très rapide.

Avec cette méthode il est permis de croire que nous pourrons mener à bien l'étude des ondes de Rayleigh pour des modèles de cristaux plus réalistes.
\end{abstract}

\begin{abstract}
We study the surface vibration modes (Rayleigh waves) by a ( moments method ) associated with state density variation models based on delta functions, for a monoatomic cubic crystal in two cases:

- first in the case of two free surfaces, taking account of first and second neighbours interactions, for a rotationally invariant crystal model, isotropic in the limit of large wavelengths ;

- second in the case of an adsorbed atom layer, considering that only the surface atoms mass changes, for a simplified model of translationally invariant crystal model with first neighbours interactions.

Comparing our results with those obtained with a more sophisticated phase shift method, we find the same phonon dispersion curve with a precision of $5 \%$ in the less good cases and $1 \%$ otherwise, with the advantage of a very rapid and analytic method. With this method, it is allowed to believe that we will get more results for the theoretical atomic studies of Rayleigh waves in more realistic crystal models.
\end{abstract}

Introduction. - L'étude des propriétés vibrationnelles des surfaces des solides cristallins a été entreprise par de nombreux auteurs, et notamment par Lifschitz et al. [1].

La méthode des fonctions de Green a été appliquée par Rosenzweig [2] à un modèle de cristal cubique simple, et lui a permis de retrouver, dans le cas d'un cristal isotrope à la limite des grandes longueurs d'ondes, les ondes de Rayleigh [3] pour une surface (001) dans la direction de propagation (100).

Cette méthode fut simplifiée par Dobrzynski et Leman [4] par des considérations de symétrie. Ils lui ont associé la méthode des déphasages généralisés [5], [6] pour résoudre le problème des vibrations des surfaces cristallines dans le cas d'un cristal mono-

$\left(^{*}\right)$ Ce travail est l'objet d'une thèse de $3^{\mathrm{e}}$ cycle en préparation.

(**) Equipe de Recherche du C. N. R. S., n 59. atomique cubique simple avec interactions centrales entre premiers voisins [7]. Masri et Dobrzynski [8] ont à leur tour appliqué cette méthode simplifiée des fonctions de Green à l'étude des vibrations de la surface (001) du modèle de cristal cubiqüe simple de Rosenzweig [2]. Ils ont alors retrouvé les ondes de Rayleigh à la limite des grandes longueurs d'ondes, et, de plus, ils ont montré l'existence d'états localisés en surface dans le «gap » șitué entre les bandes du cristal infini.

Par ailleurs, Lannoo et Lenglart [9] utilisaient la méthode des moments pour l'étude des états électroniques localisés dus aux défauts dans l'approximation des liaisons fortes. Les modèles à base de fonctions delta utilisés ont l'avantage d'apporter une très grande simplification des calculs, tout en permettant d'obtenir les résultats avec une bonne approximation. 
Nous nous proposons d'appliquer cette méthode des moments à l'étude des phonons localisés de surface, en vue de retrouver les ondes de Rayleigh de façon très rapide.

Après avoir indiqué la méthode suivie, nous envisagerons deux cas :

$1^{\mathrm{e}^{\mathrm{r}}}$ cas : étude des surfaces libres, dans le modèle de surface de Lifschitz [1].

$2^{\mathrm{e}}$ cas : étude d'une couche plane d'atomes adsorbés, de masse différente de celle des atomes du cristal, dans un modèle de Montroll-Potts [7].

Nous comparerons les résultats obtenus par cette méthode des moments à ceux fournis par la méthode des déphasages.

II. Méthode des moments. - 2.1 Matrice DYNAMIQUE ET MOMENTS. - Les équations du mouvement des atomes d'un cristal s'écrivent dans l'approximation harmonique sous la forme matricielle :

$$
\left(\mathrm{D}-\omega^{2} \mathrm{I}\right) \mathbf{u}=0
$$

D est la matrice dynamique, dont les éléments sont déterminés par les constantes de forces intervenant dans le cristal envisagé, $\omega$ la fréquence angulaire de vibration d'un atome, $I$ la matrice unité, u le vecteur déplacement, à $3 N$ composantes, des $N$ atomes du cristal par rapport à leur position d'équilibre.

Notons $\mathrm{D}_{0}$ la matrice dynamique associée à un cristal parfait infini constitué d'atomes de masse $M$, et $\mathrm{D}$ la matrice dynamique associée au cristal perturbé. Nous écrivons

$$
\mathrm{D}=\mathrm{D}_{0}+\mathrm{V}
$$

où $\mathrm{V}$ est la matrice de perturbation introduite.

Définissant les moments $\mu_{k}^{0}$ et $\mu_{k}$ comme les traces respectives des matrices $\mathrm{D}_{0}^{k}$ et $\mathrm{D}^{k}$ [10], nous pouvons définir leurs variations dues à la perturbation $\mathrm{V}$ par :

$$
\delta \mu_{k}=\mu_{k}-\mu_{k}^{0}=\operatorname{Tr}\left(\mathrm{D}^{k}\right)-\operatorname{Tr}\left(\mathrm{D}_{0}^{k}\right)
$$

et en particulier nous aurons, en fonction de $\mathrm{D}_{0}$ et $\mathrm{V}$ :

$$
\begin{aligned}
\delta \mu_{1} & =\operatorname{Tr}(\mathrm{D})-\operatorname{Tr}\left(\mathrm{D}_{0}\right)=\operatorname{Tr}(\mathrm{V}) \\
\delta \mu_{2} & =\operatorname{Tr}\left(\mathrm{D}^{2}\right)-\operatorname{Tr}\left(\mathrm{D}_{0}^{2}\right) \\
& =2 \operatorname{Tr}\left(\mathrm{VD}_{0}\right)+\operatorname{Tr}\left(\mathrm{V}^{2}\right) \\
\delta \mu_{3} & =\operatorname{Tr}\left(\mathrm{D}^{3}\right)-\operatorname{Tr}\left(\mathrm{D}_{0}^{3}\right) \\
& =3 \operatorname{Tr}\left(\mathrm{VD}_{0}^{2}\right)+3 \operatorname{Tr}\left(\mathrm{V}^{2} \mathrm{D}_{0}\right)+\operatorname{Tr}\left(\mathrm{V}^{3}\right) .
\end{aligned}
$$

2.2 ETATS LOCALisÉS 《SORTANT 》 D'UNE BANDE (Modèles EN FONCTIONS DELTA). - Considérons le problème d'un état localisé provenant d'une bande et dû à un défaut, en supposant que le nombre d'états ne change pas lorsque l'on passe du cristal parfait au cristal perturbé.

S'il y a création d'un état lié, le nombre total d'états " perdus » dans la bande est nécessairement égal à un.
Premier modèle. - Pour simplifier, nous représenterons $\delta n(E)$ dans la bande par $-\delta\left(E-E_{0}\right)$, l'état lié se trouvant à l'énergie $E_{\mathrm{L}}$ (Fig. 1).

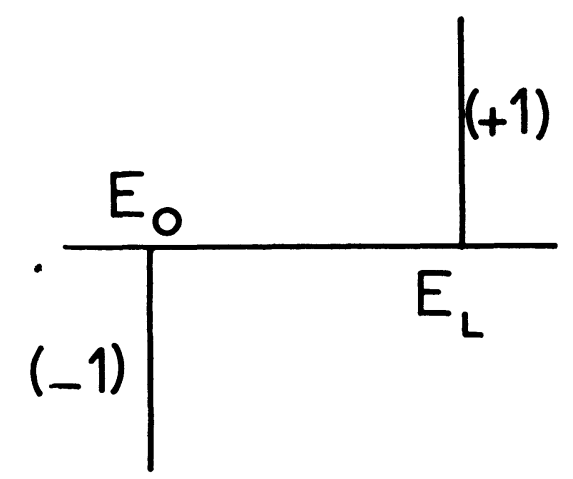

FIG. 1. - Premier modèle en fonctions delta.

Dans un modèle à deux moments, en posant dans l'équation (1)

$$
E_{\mathrm{L}}=\omega_{\mathrm{L}}^{2}
$$

nous aurons, d'après éq. 4.1 et 4.2 .

$$
\begin{aligned}
& \delta \mu_{1}=\operatorname{Tr}(\mathrm{D})-\operatorname{Tr}\left(\mathrm{D}_{0}\right)=E_{\mathrm{L}}-E_{0} \\
& \delta \mu_{2}=\operatorname{Tr}\left(\mathrm{D}^{2}\right)-\operatorname{Tr}\left(\mathrm{D}_{0}^{2}\right)=E_{\mathrm{L}}^{2}-E_{0}^{2}
\end{aligned}
$$

soit, après résolution :

$$
E_{\mathrm{L}}=\frac{1}{2}\left(\delta \mu_{1}+\frac{\delta \mu_{2}}{\delta \mu_{1}}\right) \text {. }
$$

Nous verrons que ce modèle ne sera pas satisfaisant pour des ondes de Rayleigh, car à la limite des grandes longueurs d'ondes la courbe de dispersion ne passe pas par l'origine. Nous envisagerons donc un second modèle.

Second modèle, à 2 moments. - Ce modèle va permettre un ajustement (physique) de la courbe de dispersion à. l'origine. Nous supposons que deux états $E_{1}$ et $E_{2}$ de la bande ont une contribution égale dans l'apparition d'un état localisé $E_{\mathrm{L}}$. Nous leur attribuons les poids respectifs $-\frac{1}{2},-\frac{1}{2},+1$, suivant la figure 2 .

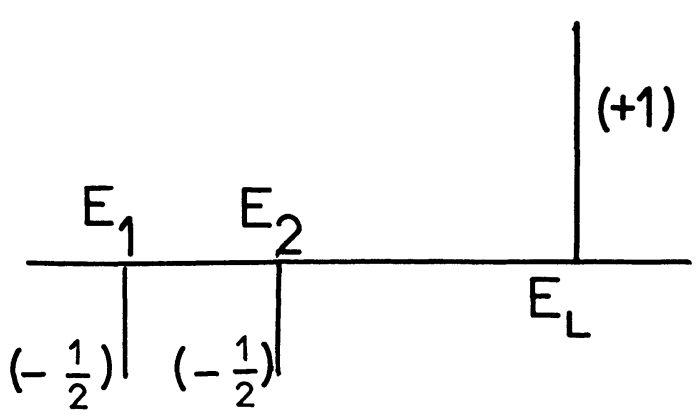

FIG. 2. - Second modèle en fonctions delta (entre parenthèses, les « poids » des fonctions). 
La différence $\left|E_{2}-E_{1}\right|$ sera le paramètre d'ajustement.

Dans un modèle à 2 moments nous aurons :

$$
\begin{aligned}
& \delta \mu_{1}=E_{\mathrm{L}}-\frac{1}{2} E_{1}-\frac{1}{2} E_{2} \\
& \delta \mu_{2}=E_{\mathrm{L}}^{2}-\frac{1}{2} E_{1}^{2}-\frac{1}{2} E_{2}^{2} .
\end{aligned}
$$

Posant

$$
\begin{aligned}
E_{1} & =\frac{1}{2}\left(E_{1}+E_{2}\right)+\frac{1}{2}\left(E_{1}-E_{2}\right) \\
& =E_{\mathrm{L}}-\delta \mu_{1}+\frac{1}{2}\left(E_{1}-E_{2}\right) .
\end{aligned}
$$

Nous éliminons $E_{L}^{2}$ et trouvons :

$$
E_{\mathrm{L}}=\frac{1}{2}\left[\delta \mu_{1}+\frac{\delta \mu_{2}}{\delta \mu_{1}}+\frac{1}{\delta \mu_{1}}\left(\frac{E_{2}-E_{1}}{2}\right)^{2}\right]
$$

le paramètre $\left(E_{2}-E_{1} / 2\right)^{2}$ devant être déterminé de telle façon que la courbe de dispersion passe par l'origine.

Troisième modèle, à 3 moments. - Le même modèle, utilisé avec 3 moments, conduit aux équations suivantes :

$$
\begin{aligned}
& \delta \mu_{1}=E_{\mathrm{L}}-\frac{1}{2}\left(E_{1}+E_{2}\right) \\
& \delta \mu_{2}=E_{\mathrm{L}}^{2}-\frac{1}{2} E_{1}^{2}-\frac{1}{2} E_{2}^{2} \\
& \delta \mu_{3}=E_{\mathrm{L}}^{3}-\frac{1}{2} E_{1}^{3}-\frac{1}{2} E_{2}^{3}
\end{aligned}
$$

Par élimination entre ces 3 équations nous aboutissons à l'équation en $E_{\mathrm{L}}$ :

$$
E_{\mathrm{L}}^{2}-E_{\mathrm{L}}\left(2 \delta \mu_{1}+\frac{\delta \mu_{2}}{\delta \mu_{1}}\right)+\frac{2}{3} \delta \mu_{1}^{2}+\delta \mu_{2}+\frac{1}{3} \frac{\delta \mu_{3}}{\delta \mu_{1}}=0
$$

Cette équation admet généralement deux solutions dont l'une est à rejeter en vertu de considérations physiques (par exemple $E_{\mathrm{L}}<0$ ).

III. Vibrations localisées de surfaces libres. PrÉSENTATION DU MODÈLE. - Nous utilisons un modèle de cristal cubique simple, formé d'atomes identiques de masse $M$.

Pour obtenir deux surfaces libres, nous coupons le réseau infini en deux par le plan $z=a / 2$, où $a$ est le paramètre cristallin, et nous supprimons les interactions entre les atomes se trouvant de part et d'autre de ce plan.

Dans ce modèle nous supposons que les positions d'équilibre des atomes de la surface libre sont les mêmes que celles du cristal infini, et aussi que les forces de liaison interatomiques sont inchangées en surface. Ce modèle est invariant par translation du réseau comme l'ont remarqué Lifschitz et al. [1].

Nous tenons compte par ailleurs des interactions centrales entre premiers et seconds voisins, ainsi que des forces dues à la rigidité angulaire d'un système de trois premiers voisins consécutifs formant un angle droit à l'équilibre. Ce modèle satisfait à la fois les conditions de la stabilité élastique

$$
\begin{array}{r}
\left(\mathrm{C}_{11}>0, \mathrm{C}_{44}>0, \mathrm{C}_{11}-\mathrm{C}_{12}>0, \mathrm{C}_{11}+2 \mathrm{C}_{12}>0\right) \\
-\mathrm{C}_{12}
\end{array}
$$

et les conditions d'invariance de l'énergie potentielle lors d'une rotation infinitésimale de l'ensemble du cristal [11].

Matrice dynamique. Cristal non Perturbé. Désignons par $\mathbf{u}_{1}$ le déplacement de l'atome placé au site $\mathbf{R}_{\mathbf{l}}$. Nous savons que

$$
\mathbf{R}_{1}=l_{1} \mathbf{a}_{1}+l_{2} \mathbf{a}_{2}+l_{3} \mathbf{a}_{3}
$$

où $\mathbf{a}_{i}$ sont les vecteurs de base, de module $a$, du réseau cubique, et $l=\left(l_{1}, l_{2}, l_{3}\right)$ avec $l_{i}$ entiers. Nous noterons

$$
u_{1}^{\sigma} \quad \text { avec } \quad \sigma=x, y \text { ou } z
$$

les composantes suivant les 3 axes du vecteur déplacement.

Pour une surface (001) désignée par $l_{3}=\mathrm{Cte}$, nous pouvons poser, du fait de l'existence de la symétrie de translation dans le plan de la surface :

$u_{1}^{\sigma}=\frac{1}{M^{1 / 2}} v_{1_{3}}^{\sigma}\left(\varphi_{1}, \varphi_{2}\right) \exp \left[i\left(1_{1} \varphi_{1}+1_{2} \varphi_{2}-\omega t\right)\right]$

où $\varphi=\left(\varphi_{1}, \varphi_{2}, \varphi_{3}\right)$ est le vecteur :

$$
\varphi=a \mathbf{k}
$$

$\mathbf{k}=\left(\mathrm{k}_{1}, \mathrm{k}_{2}, \mathrm{k}_{3}\right)$ étant le vecteur de propagation, et $\omega$ la fréquence angulaire, $t$ le temps, $M$ la masse.

Notons respectivement $\chi_{1}, \chi_{3}$ et $\chi_{5}$ les rapports sur $M$, des constantes de forces centrales entre premiers voisins, seconds voisins et non centrales dues à la rigidité angulaire d'un système de trois premiers voisins formant un angle droit à l'équilibre. Dans l'approximation d'un cristal isotrope aux grandes longueurs d'ondes, nous avons les relations entre ces constantes : $\chi_{1}=2 \chi_{3} ; \chi_{3}=\chi_{5}$.

Alors, les équations du mouvement se mettent sous la forme matricielle suivante, en tenant compte de (12):

$$
\left[\mathrm{D}_{0}\left(\varphi_{1}, \varphi_{2}\right)-\omega^{2}\left(\varphi_{1}, \varphi_{2}\right) \mathrm{I}\right] V_{1_{3}}=0
$$

où $D_{0}\left(\varphi_{1}, \varphi_{2}\right)$ est la matrice dynamique du tableau 1 .

SimplificATION DU PROBlèME. - La matrice $\mathrm{D}_{0}$ est représentée par rapport à une base de fonctions du type $\left|l_{3}, \sigma\right\rangle$, en utilisant cette notation condensée pour montrer la dépendance en $l_{3}$ et $\sigma$ dans (12). Nous nous intéressons aux plans des surfaces libres, donc à ceux pour lesquels $l_{3}=0$ ou $1, \sigma=x$ ou $y$ ou $z$.

L'étude de la symétrie faite pour la zone de Brillouin à 2 dimensions associée à la surface [4], figure 3, a montré que l'on pouvait prendre comme base des 


\section{TABLEAU I}

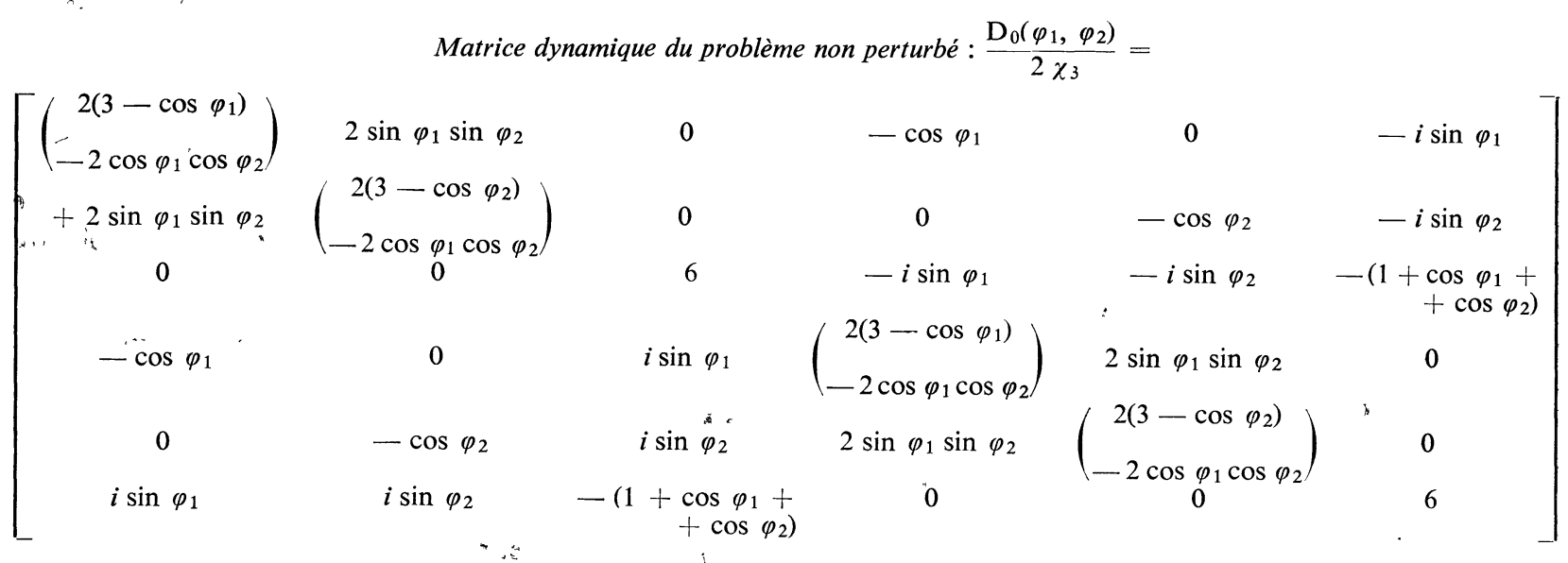

représentations irréductibles les fonctions suivantes, $|0, x\rangle-|1, x\rangle|0, y\rangle-|1, y\rangle$ au facteur près $1 / \sqrt{2}$ de normalisation :

$$
|0, z\rangle+|1, z\rangle
$$

$|0, x\rangle+|1, x\rangle|0, y\rangle+|1, y\rangle$

$$
|0, z\rangle-|1, z\rangle
$$

La matrice dynamique s'écrit alors après changement de base sous la forme de blocs :

$$
\begin{aligned}
& D_{0}=2 \chi_{3}\left(\begin{array}{cc}
D_{01} & 0 \\
0 & D_{02}
\end{array}\right)
\end{aligned}
$$

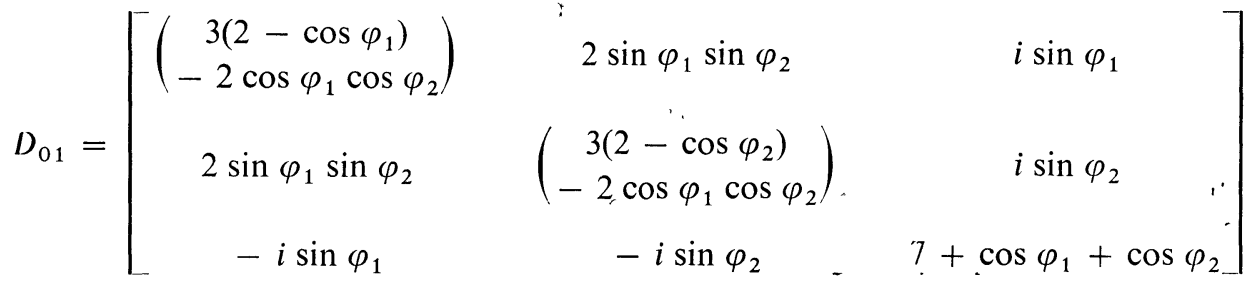

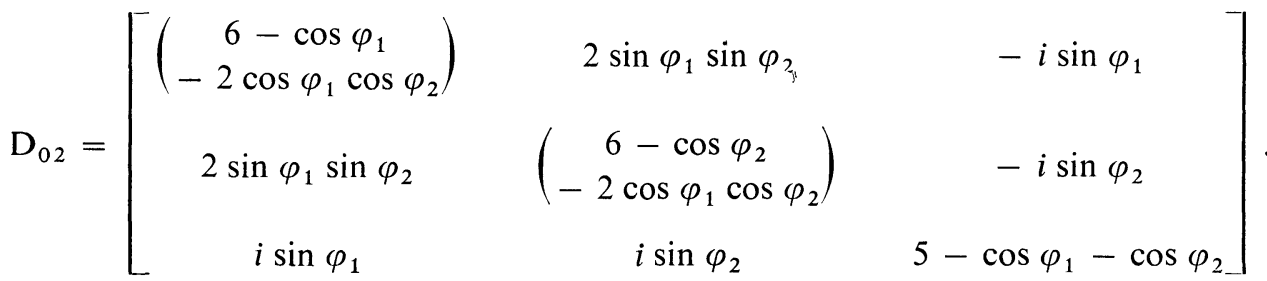

$$
\begin{aligned}
& \mathrm{D}=2 \chi_{3}\left(\begin{array}{cc}
\mathrm{D}_{1} & 0 \\
0 & \mathrm{D}_{2}
\end{array}\right)
\end{aligned}
$$

permet de présenter la matrice dynamique du problème perturbé sous la forme simple suivante :

avec

$$
\mathrm{D}_{1}=\left[\begin{array}{ccc}
\left(\begin{array}{c}
5-2 \cos \varphi_{1} \\
-2 \cos \varphi_{1} \cos \varphi_{2}
\end{array}\right) & 2 \sin \varphi_{1} \sin \varphi_{2} & 0 \\
2 \sin \varphi_{1} \sin \varphi_{2} & \left(\begin{array}{c}
5-2 \cos \varphi_{2} \\
-2 \cos \varphi_{1} \cos \varphi_{2}
\end{array}\right) & 0 \\
0 & 0 & 3
\end{array}\right]
$$

et

$$
\begin{aligned}
& \mathrm{D}_{2}= \mathrm{D}_{1} \\
& \mathrm{~V}=\mathrm{D}-\mathrm{D}_{0}=-2 \chi_{3}\left(\begin{array}{cc}
\mathrm{V}^{\mathrm{S}} & 0 \\
0 & \mathrm{~V}^{\mathrm{AS}}
\end{array}\right)
\end{aligned}
$$




$$
\begin{aligned}
\mathrm{V}^{\mathrm{S}} & =\left[\begin{array}{ccc}
1-\cos \varphi_{1} & 0 & i \sin \varphi_{1} \\
0 & 1-\cos \varphi_{1} & i \sin \varphi_{2} \\
-i \sin \varphi_{1} & -i \sin \varphi_{2} & 4+\cos \varphi_{1}+\cos \varphi_{2}
\end{array}\right] \\
\mathrm{V}^{\mathrm{AS}} & =\left[\begin{array}{ccc}
1+\cos \varphi_{1} & 0 & -i \sin \varphi_{1} \\
0 & 1+\cos \varphi_{2} & -i \sin \varphi_{2} \\
i \sin \varphi_{1} & i \sin \varphi_{2} & 2-\cos \varphi_{1}-\cos \varphi_{2}
\end{array}\right] .
\end{aligned}
$$

CAlCUl DES ÉTATS LOCALISÉS. - Nous sommes à présent en mesure de calculer les variations des moments par les formules (4) ainsi que les états localisés par (7), (9), (11). Etant donné que les matrices sont décomposées en somme directe suivant une partie dite symétrique (S) et une autre antisymétrique (AS) on peut décomposer le calcul des traces en deux parties, et on obtient pour les moments :

$$
\begin{aligned}
\delta \mu_{1}^{\mathrm{S}} & =-12 \chi_{3} \\
\delta \mu_{1}^{\mathrm{AS}} & =-8 \chi_{3} \\
\delta \mu_{1} & =-20 \chi_{3} \\
\delta \mu_{2}^{\mathrm{S}} & =8 \chi_{3}^{2}\left[-33+2\left(\cos \varphi_{1}+\cos \varphi_{2}\right)+3 \cos \varphi_{1} \cos \varphi_{2}-2\left(\cos ^{2} \varphi_{1}+\cos ^{2} \varphi_{2}\right)+\right. \\
\delta \mu_{2}^{\mathrm{AS}} & =8 \chi_{3}^{2}\left[-17+3 \cos \varphi_{1} \cos \varphi_{2}-2\left(\sin ^{2} \varphi_{1}+\sin ^{2} \varphi_{2}\right)+2 \cos \varphi_{1} \cos \varphi_{2}\left(\cos \varphi_{1}+\cos \varphi_{2}\right)\right] \\
\delta \mu_{2} & \left.=16 \chi_{3}^{2}\left[-27+\cos \varphi_{1}+\cos \varphi_{2}\right)\right]
\end{aligned}
$$

etc...

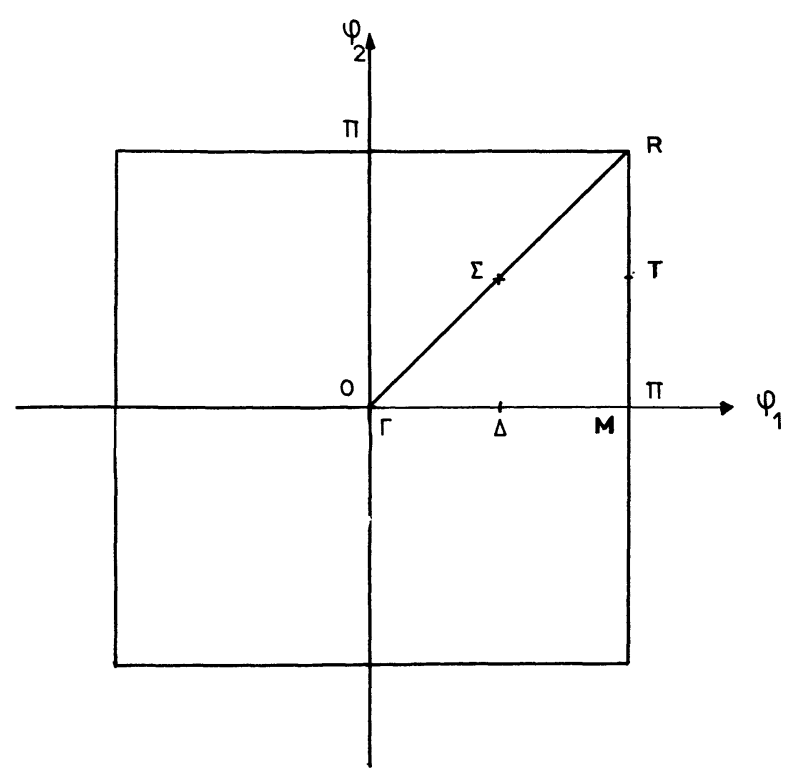

FIg. 3. - Zone de Brillouin à 2 dimensions, associée à la face (001) d'un cristal cubique simple.

Le calcul effectué pour le modèle de cristal [8] montre que la fréquence maximale de vibration est telle que

$$
\omega_{M}^{2}=24 \chi_{3} .
$$

Les équations (5) et (20) permettent donc de calculer les rapports $\omega_{\mathrm{L}} / \omega_{\mathrm{M}}$.
Nous donnons ci-dessous les résultats pour les trois modèles.

Modèle 1 (à 2 moments). - Les résultats sont portés sur la figure 4 .

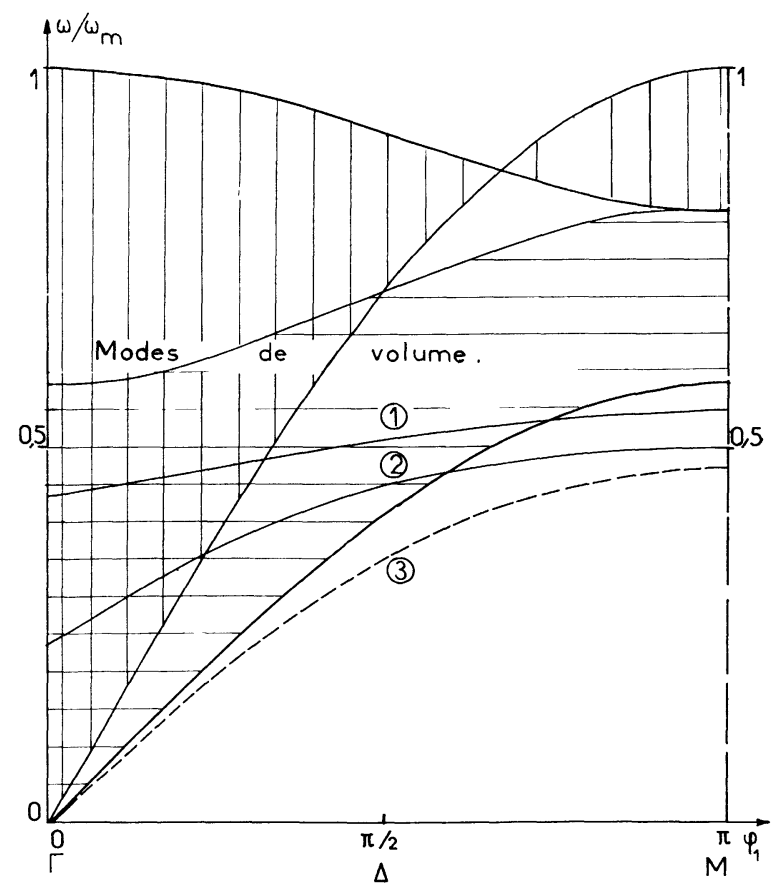

FIG. 4. - Courbes de dispersion des phonons localisés, pour le premier modèle avec fonctions delta : 1. Cas $S$; 2. Cas AS ; 3. Calculs, réf. [8] par la méthode des déphasages. 


\section{TABLEAU II}

Résultats du premier modèle avec fonctions delta, comparés à ceux d'un calcul par la méthode des déphasages.

$\begin{array}{cccc}\omega_{\mathrm{L}} / \omega_{\mathrm{M}} & \text { Cas S } & \text { Cas AS } & \begin{array}{c}\text { Calcul } \\ \text { réf. [8] }\end{array} \\ - & - & - & \overline{0} \\ \varphi_{1}=\bar{\varphi}_{2}=0 & 0,440 & 0,24 & 0 \\ \varphi_{1}=\pi \varphi_{2}=0 & 0,554 & 0,50 & 0,475\end{array}$

Nous voyons que ce modèle est intéressant dans la mesure où il permet d'espérer voir " sortir " de la bande des états localisés. Il est cependant insuffisant, car les courbes de dispersion des phonons ne passent pas par l'origine.

Modèle 2 (à 2 moments, avec ajustement). - En imposant $E_{\mathrm{L}}=0$ pour $\varphi_{1}=\varphi_{2}=0$ on trouve

$$
\left(\frac{E_{2}-E_{1}}{2}\right)^{2}\left\{\begin{array}{l}
=112 \chi_{3}^{2} \text { dans le cas } \mathrm{S} ; \\
=16 \chi_{3}^{2} \text { dans le cas AS } .
\end{array}\right.
$$

\section{TABleau III}

Résultats du second modèle, comparés à ceux d'un calcul par la méthode des déphasages

$\begin{array}{cccc}\omega_{\mathrm{L}} / \omega_{\mathrm{M}} & \text { Cas S } & \text { Cas AS } & \begin{array}{c}\text { Calcul } \\ \text { réf. [8] } \\ \text { Masri et al. }\end{array} \\ \varphi_{1}=\overline{\varphi_{2}}=0 & \overline{0} & \overline{0} & \overline{0} \\ \varphi_{1}=\pi, \varphi_{2}=0 & 0,478 & 0,456 & 0,475\end{array}$

Les résultats sont portés sur la figure 5 .

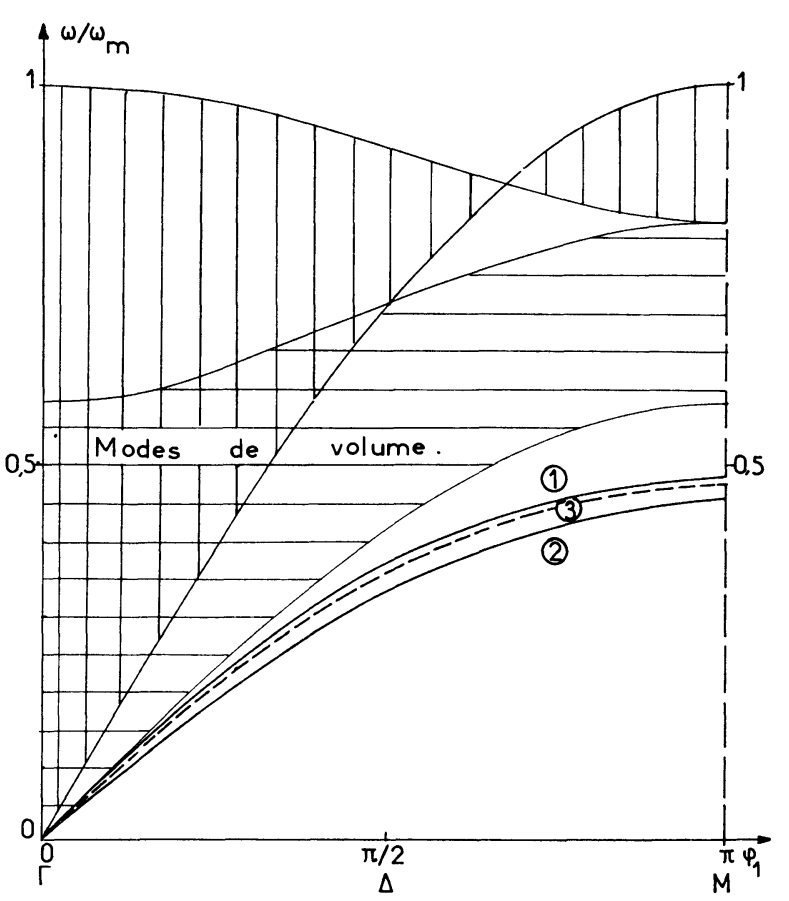

FIG. 5. - Courbe de dispersion des phonons localisés pour le second modèle. 1. Cas $S ; 2$. Cas AS ; 3. Calculs réf. [8].
L'erreur faite en $\varphi_{1}=\pi \varphi_{2}=0$ est inférieure à $5 \%$ dans le cas AS et $0,5 \%$ dans le cas $S$.

Nous voyons donc que ce modèle, malgré sa simplicité et la rapidité des calculs fournit une très bonne valeur des ondes de Rayleigh. Son principal désavantage est de nécessiter un ajustement ; c'est pourquoi nous essayons le troisième modèle.

Modèle 3 (à 3 moments), - Ce modèle ne nécessite ici aucun ajustement. Seules les valeurs des moments sont plus longues à obtenir.

\section{TABleaU IV}

Résultats du troisième modèle, comparés à ceux d'un calcul par la méthode des déphasages

$\begin{array}{cccc}\omega_{\mathrm{L}} / \omega_{\mathrm{M}} & \text { Cas S } & \text { Cas AS } & \begin{array}{c}\text { Calcul } \\ \text { réf. [8] } \\ \text { Masri et al. }\end{array} \\ \varphi_{1}=\varphi_{2}=0 & \overline{0} & \overline{0} & - \\ \varphi_{1}=\pi, \varphi_{2}=0 & 0,480 & 0,477 & 0,475\end{array}$

Les courbes de dispersion sont alors très voisines l'une de l'autre : l'erreur est inférieure à $0,5 \%$.

Conclusion. - Nous voyons que ces modèles donnent pour les ondes de Rayleigh des résultats de plus en plus voisins des résultats exacts obtenus par la méthode analytique des fonctions de Green. Cette constatation est assez logique étant donné que les modèles choisis sont de plus en plus "fins ». Il faut remarquer cependant que le modèle 3 , qui utilise trois moments, donne dans le cas présent des résultats très précis par une méthode rapide, et sans nécessiter un ajustement à l'origine de la courbe de dispersion.

La méthode des moments associée à ces modèles aux fonctions delta est donc encourageante pour l'étude des vibrations des surfaces cristallines libres.

IV. Vibrations localisées d'une couche d'atomes adsorbés. - PRÉSENTATION DU MODÈLE - Etudions maintenant le cas d'une couche plane d'atomes adsorbés en prenant le modèle de cristal de MontrollPotts [7], déjà utilisé par ces auteurs dans l'étude des défauts ponctuels à l'intérieur des cristaux.

Il s'agit d'un modèle de cristal cubique simple d'atomes de masse $M$, distants de $a$, avec interactions centrales entre premiers voisins.

La couche d'atomes adsorbés est simulée par la couche extrême du demi-cristal, où seule la masse des atomes est changée. Nous supposons que la position d'équilibre d'un atome adsorbé est la même que celle qu'occuperait un atome du cristal parfait infini, et que les constantes de forces ont la même valeur en surface et à l'intérieur du cristal.

Il est bien connu que ce modèle est en désaccord avec la théorie de l'élasticité, et que les conditions d'invariance de l'énergie potentielle lors d'une rotation infinitésimale du solide ne sont pas satisfaites [11]. 
Néanmoins la symétrie de translation le long de la surface est conservée et on peut s'attendre avec ce modèle à obtenir une bonne approximation des grandeurs physiques [12]-[13]. Nous traitons encore comme une perturbation la présence d'une couche adsorbée.

Notons encore qu'une augmentation de la constante de force en surface peut être simulée physiquement par une diminution de la masse des atomes adsorbés, et inversement.
MATRICES DYNAMIQUeS ET DE PERTURBation. Nous procédons comme en II pour obtenir les équations du mouvement (éq. 13). Appelant $\beta$ la constante de force entre premiers voisins, $\boldsymbol{M}$ la masse des atomes du cristal infini et $M^{\prime}$ la masse des atomes adsorbés nous aurons alors, en conservant les notations précédentes :

- Matrice dynamique du cristal non perturbé :

$$
\mathrm{D}_{0}\left(\varphi_{1}, \varphi_{2}\right)=\frac{\beta}{M}\left[\begin{array}{ccc}
5-2 \cos \varphi_{1}-2 \cos \varphi_{2} & -1 & 0 \\
-1 & 6-2 \cos \varphi_{1}-2 \cos \varphi_{2} & -1 \\
0 & -1 & 6-2 \cos \varphi_{1}-2 \cos \varphi_{2}
\end{array}\right]
$$

Matrice du cristal perturbé :

$$
\mathrm{D}\left(\varphi_{1}, \varphi_{2}\right)=\beta\left[\begin{array}{ccc}
\frac{1}{M^{\prime}}\left(5-2 \cos \varphi_{1}-2 \cos \varphi_{2}\right) & -\frac{1}{M^{\prime}} & 0 \\
-\frac{1}{M} & \frac{1}{M}\left(6-2 \cos \varphi_{1}-2 \cos \varphi_{2}\right) & -\frac{1}{M} \\
0 & -\frac{1}{M} & \frac{1}{M}\left(6-2 \cos \varphi_{1}-2 \cos \varphi_{2}\right)
\end{array}\right] .
$$

Matrice de Perturbation :

$$
\mathrm{V}=\mathrm{D}-\mathrm{D}_{0}=\beta\left(\frac{1}{M^{\prime}}-\frac{1}{M}\right)\left[\begin{array}{crc}
5-2 \cos \varphi_{1}-2 \cos \varphi_{2} & -1 & 0 \\
0 & 0 & 0 \\
0 & 0 & 0
\end{array}\right] .
$$

Moments ET ÉTATS LOCALiSÉS. - Le calcul des moments $\delta \mu_{k}$ s'effectue par (4.1) et (4.2).

$$
\begin{aligned}
& \delta \mu_{1}=\beta\left[\frac{1}{M^{\prime}}-\frac{1}{M}\right]\left(5-2 \cos \varphi_{1}-2 \cos \varphi_{2}\right) \\
& \delta \mu_{2}=\beta^{2}\left(\frac{1}{M^{\prime}}-\frac{1}{M}\right)\left[\left(5-2 \cos \varphi_{1}-2 \cos \varphi_{2}\right)^{2}\left(\frac{1}{M^{\prime}}+\frac{1}{M}\right)+\frac{2}{M}\right] .
\end{aligned}
$$

Pour simplifier, nous effectuons les changements de et donc la valeur maximum de $\omega^{2}$ est : variables

$$
\begin{aligned}
& X=3-\cos \varphi_{1}-\cos \varphi_{2} \\
& \alpha=\frac{M^{\prime}-M}{M} .
\end{aligned}
$$

Alors nous obtenons :

$$
\begin{aligned}
& \delta \mu_{1}=\frac{\beta}{M} \frac{-\alpha}{1+\alpha}(2 X-1) \\
& \delta \mu_{2}=\left(\frac{\beta}{M}\right)^{2} \frac{-\alpha}{1+\alpha}\left[\frac{2+\alpha}{1+\alpha}(2 X-1)^{2}+2\right] .
\end{aligned}
$$

\section{Rappelons [12] que}

$\omega^{2}(\mathrm{k})=2 \frac{\beta}{M}\left(3-\cos \varphi_{1}-\cos \varphi_{2}-\cos \varphi_{3}\right)$

$$
\omega_{M}^{2}=12 \frac{\beta}{M}
$$

Nous allons présenter les résultats donnant $\omega_{\mathrm{L}} / \omega_{M}$ dans les cas $M^{\prime}=M / 2$ et $M^{\prime}=2 M$, pour les points de la zone de Brillouin situés sur le chemin $(\Gamma M R \Gamma)$. (Fig. 3), en vue de comparer nos valeurs à celles de la référence [12].

AtOMES ADSORBÉS LOURDS. $-M^{\prime}=2 M, \alpha=1$ (voir Fig. 6).

Premier modèle (à deux moments) (7). - Il est intéressant dans la mesure où il permet d'espérer voir « sortir » des états localisés de la bande de volume.

Second modèle (à deux moments avec ajustement) (9). 


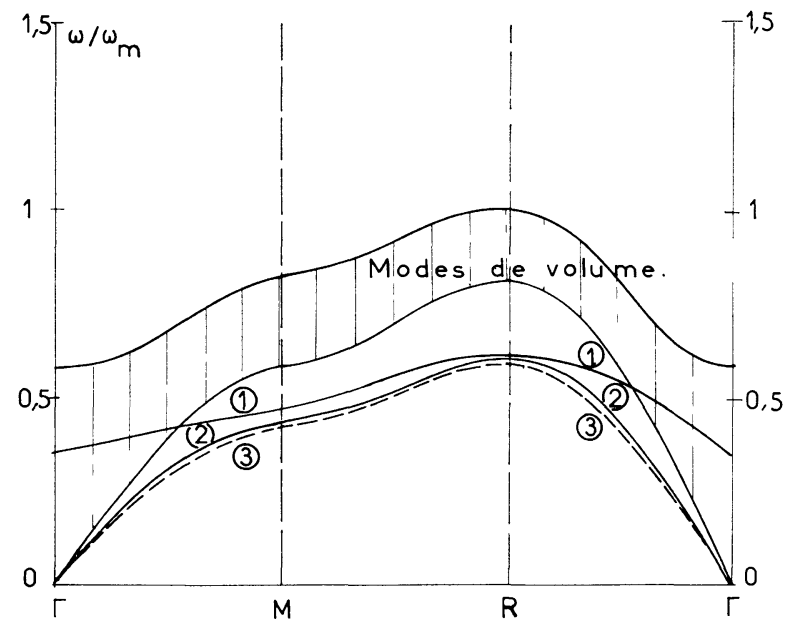

FIg. 6. - Spectre de vibrations dans le cas d'atomes adsorbés lourds, $\left(M^{\prime}=2 M\right): 1$. Premier modèle ; 2 . Second modèle ; 3. Calculs réf. [12] par la méthode des fonctions de Green. Les points indiqués correspondent aux directions de propagàtion repérées sur la zone de Brillouin (Fig. 3).

- La condition $E_{\mathrm{L}}=0$ pour $\varphi_{1}=\varphi_{2}=0$ fournit la valeur suivante :

$$
\left(\frac{E_{2}-E_{1}}{2}\right)^{2}=\frac{3}{2}\left(\frac{\beta}{M}\right)^{2}
$$

La courbe de dispersion obtenue par (9) et (29) fournit une approximation à $1 \%$ près environ par rapport à celle calculée par la méthode des fonctions de Green.

$$
\left(\frac{\omega_{L}}{\omega_{M}}\right)^{2}=\frac{1}{24}\left(2 X-1-\frac{1}{2 X-1}\right) \text {. }
$$

Ce même modèle fournit, dans le cas d'une couche d'atomes adsorbés lourds $\left(M^{\prime}>M\right)(\alpha$ positif) la relation :

$$
\left(\frac{\omega_{\mathrm{L}}}{\omega_{\mathrm{M}}}\right)^{2}=\frac{1}{12(1+\alpha)}\left(2 X-1-\frac{1}{2 X-1}\right) .
$$

Si $M^{\prime}$ augmente, $\alpha$ augmente, et la courbe de dispersion s'aplatit sur l'axe des abscisses, ce à quoi on peut s'attendre physiquement.

ATOMES ADSORBÉS LÉGERS.

$$
M^{\prime}=\frac{M}{2}, \quad \alpha=-\frac{1}{2} . \quad \text { (Voir Fig. 7.) }
$$

Second modèle. - Ici nous devons effectuer l'ajustement en haut de bande car les fréquences localisées sont supérieures aux fréquences de la bande de volume [12]. Pour $\varphi_{1}=\varphi_{2}=0$ on a :

$$
\left(\frac{\omega_{\mathrm{L}}}{\omega_{\mathrm{M}}}\right)^{2}=\frac{1}{3}
$$

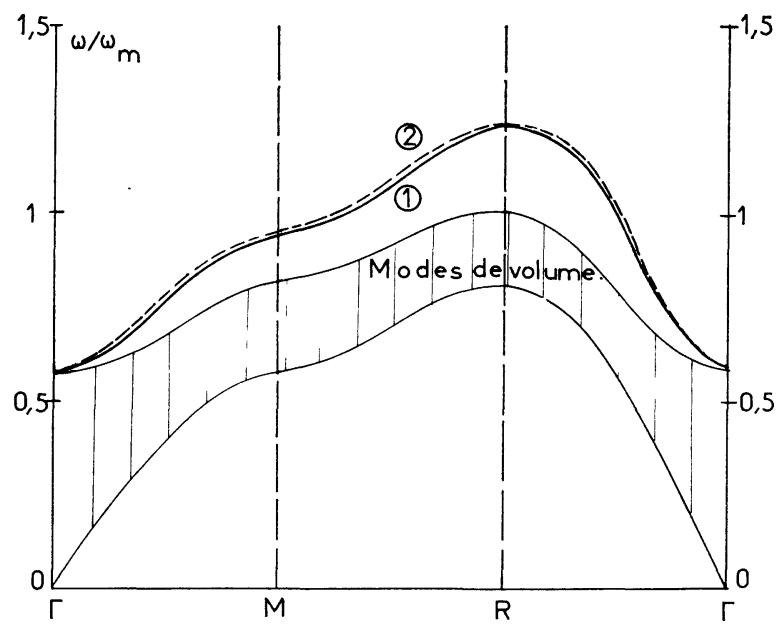

FIG. 7. - Spectre de vibrations dans le cas d'atomes adsorbés légers $\left(M^{\prime}=M / 2\right): 1$. Second modèle ; 2. Calculs réf. [12]. Les points indiqués correspondent aux directions de propagation repérées sur la zone de Brillouin (Fig. 3).

Cette valeur portée dans l'équation $E_{\mathrm{L}}=0$ (éq. 9) fournit pour l'ajustement :

$$
\left(\frac{E_{2}-E_{1}}{2}\right)^{2}=2 \text {. }
$$

Nous voyons sur la figure 7 que la courbe de dispersion des états localisés en surface a une meilleure concordance encore que dans le cas des atomes adsorbés lourds. (Au point $\mathrm{R}$ elles sont d'ailleurs confondues.)

$$
\left(\frac{\omega_{\mathrm{L}}}{\omega_{\mathrm{M}}}\right)^{2}=\frac{1}{6}\left(2 X-1+\frac{1}{2 X-1}\right) \text {. }
$$

Pour $M^{\prime}<M,(\alpha<0)$ nous aurons en général

$$
\left(\frac{\omega_{\mathrm{L}}}{\omega_{\mathrm{M}}}\right)^{2}=\frac{1}{12}\left[\frac{1}{1+\alpha}(2 X-1)-\frac{1}{\alpha} \frac{1}{2 X-1}\right] \text {. }
$$

Pente a L'origine. - Pour l'étude de la vitesse des ondes de surface, il est intéressant d'étudier la pente de la courbe de dispersion à l'origine. Pour les modes de volume, nous avons en tenant compte que $\varphi_{3}=0$ dans (28), et par (25)

$$
\left(\frac{\omega}{\omega_{M}}\right)_{\mathrm{vol}}^{2}=\frac{1}{6}(X-1) .
$$

En vue de poursuivre la comparaison avec les calculs exacts par les fonctions de Green, dans le cas $M^{\prime}=2 M$, nous faisons par ailleurs un développement de (30) en fonction de $(X-1)$ comme infiniment petit ; et nous obtenons pour les états localisés :

$$
\left(\frac{\omega_{L}}{\omega_{M}}\right)^{2} \# \frac{1}{6}(X-1)-\frac{1}{6}(X-1)^{2} \text {. }
$$


Posant enfin

$$
\left\{\begin{array}{l}
\varphi^{2}=\varphi_{1}^{2}+\varphi_{2}^{2} \\
X-1 \# \frac{1}{2} \varphi^{2}
\end{array}\right.
$$

nous aurons par différence :

$$
\left(\frac{\omega}{\omega_{M}}\right)_{\text {vol }}^{2}-\left(\frac{\omega_{\mathrm{L}}}{\omega_{\mathrm{M}}}\right)^{2} \# \frac{1}{24} \varphi^{4} .
$$

Nous en déduisons que dans le cas précis qui nous intéresse les modes localisés à l'origine ne diffèrent des modes de volume en bas de bande que par des termes du second ordre en $\varphi$, ce qui est bien le résultat trouvé par la méthode des fonctions de Green [12].

Cependant, si nous étudions le cas général en développant (31), nous voyons que les termes en $\varphi^{2}$ ne s'annulent pas, et la concordance exacte avec le résultat précédent n'est plus réalisée.

Conclusion. - Tenant compte du fait que le modèle de Montroll-Potts utilisé dans le cas de la vibration de la couche adsorbée est $a$ priori très simple, nous voyons que les modèles à deux moments pour les ondes de Rayleigh sortant de la bande de volume, fournissent des valeurs des courbes de dispersion satisfaisantes, comparativement à celles obtenues par les calculs employant la méthode des fonctions de Green.

Cependant la pente à l'origine de la courbe concorde parfaitement avec les autres calculs uniquement dans le cas précis d'atomes adsorbés lourds de masse double de celle des atomes du cristal.

Ce point particulier mériterait une étude plus détaillée, en vue d'obtenir un bon accord à la fois au voisinage de l'origine et dans le reste de la zone de
Brillouin, pour des atomes adsorbés lourds de masse quelconque.

V. Conclusion générale. - L'étude des propriétés vibrationnelles des surfaces des solides cristallins par la méthode des moments associée à des modèles à base de fonctions delta apparaît finalement comme assez encourageante dans le cas des cubiques simples.

- D'une part, nous avons montré que dans le cas de deux surfaces libres étudiées par le modèle de Rosenzweig avec interactions centrales entre seconds voisins, les modèles à deux moments permettaient de mettre en évidence les courbes de dispersion des états localisés avec une précision de $5 \%$ par rapport au calcul exact, dans les cas les moins favorables.

- D'autre part, en ce qui concerne une couche d'atomes adsorbés mettant en œuvre un modèle plus simpliste (modèle de Montroll-Potts avec interactions centrales entre premiers voisins seulement), nous retrouvons les ondes de surface à $1 \%$ près par rapport au calcul exact, et la même vitesse exactement dans le cas d'atomes adsorbés de masse double des atomes du cristal.

- Il est donc permis d'espérer que l'utilisation de cette méthode qui présente le gros avantage d'être rapide à mettre en œuvre, permettra d'étudier les vibrations cristallines avec des modèles plus réalistes, ou dans le cas de cristaux qui ne sont pas des cubiques simples avec un atome par maille.

Remerciements. - Nous voudrions remercier le Dr P. Lenglart pour nous avoir stimulés lors de cette étude par de nombreuses discussions ainsi que le Dr G. Leman pour l'intérêt qu'il y a porté.

\section{Bibliographie}

[1] LifSChitz (I. M.) et Rosenzweig (L. N.), Izvest. Akad. Nauk-SSSR Série Fiz., 1948, 12, 667.

[2] Rosenzweig (L. N.), Uchenye Zapiski Hark. Gosudarst Univ. Trudy Fiz. Mat. Otdel, 1950, 2, 19.

[3] Rayleigh (L.), Proc. London Math. Society, 1887, $4,17$.

[4] Dobrzynski (L.) et Leman (G.), J. Physique, 1969, 30, 116.

[5] DE WITT (B. S.), Phys. Rev., 1956, 103, 1565, section III.

[6] Toulouse (G.), Sol. State Comm., 1966, 4, 593.

[7] Montroll (E. W.) et Potts (R. B.), Phys. Rev., $1956,102,72$.
[8] MASri (P.) et Dobrzynski (L.), J. Physique, 1971, 32, 295.

[9] Lannoo (M.) et Lenglart (P.), J. Physique, 1971, $32,427$.

[10] Maradudin (A. A.), Montroll (E. W.) et Weiss (G. H.), Sol. State Phys., 1963, Suppl. 3.

[11] Ludwig (W.) et Lengeler (B.), Sol. State Comm., 1964, 2, 83.

[12] Dobrzynski (L.) et Mills (D. L.), J. Phys. Chem. Solids, 1969, 30, 1043.

[13] Dobrzynski (L.), Ann. Phys., 1969, 4, 637, Thèse Orsay. 